Laser Chem., 1999, Vol. 19, pp. 41-44

Reprints available directly from the publisher Photocopying permitted by license only
(C) 1999 OPA (Overseas Publishers Association) N.V. Published by license under the Harwood Academic Publishers imprint, part of The Gordon and Breach Publishing Group.

\title{
RESONANCE RAMAN AND OPTICAL DEPHASING STUDY OF HITCI
}

\author{
A. KUMMROW*, S. H. ASHWORTH and K. LENZ \\ Max-Born-Institut, Rudower Chaussee 6, D-12474 Berlin, Germany
}

(Received 7 April 1997)

Line shape analysis based on resonance Raman spectra of HITCI is used to determine the details of the vibrational part of the line broadening function. Forced Light Scattering with $20 \mathrm{fs}$ pulses from a Ti: sapphire laser measured optical dephasing probing with an $\mathrm{Ar}^{+}$laser. The observed response is well described by the line broadening function derived from the fluorescence line shape.

Keywords: Ultrafast spectroscopy; resonance Raman; line shape analysis; HITCI; fluorescence

Optical response of organic dyes is often discussed considering only two electronic levels. Femtosecond spectroscopy shows clear signatures of the vibrational dynamics involved. In the simplest case, the excited state potential surface is displaced along a number of normal coordinates. For such rigid molecules, transient four-wave-mixing experiments (TFWM) can be described with the cumulative expansion formalism. The fundamental quantity in this theory is a spectral density $\rho(\omega)$ which allows all experimental features to be calculated [1]. Vibrations involved in optical dynamics appear as resonance in $\rho(\omega)$, and are also Raman active. The aim of this paper is to clarify the relationship between $\rho(\omega)$ and the resonance Raman spectrum.

TFWM of HITCI (1,7-Bis-[1,3,3-trimethyl-3H-indol-2-yl]-heptamethinium iodide) in solution has recently been extensively studied (Refs. [2-4] and references therein). The resonance Raman spectrum, exciting at $627 \mathrm{~nm}$, shown in Figure 1 was obtained adsorbing the dye

\footnotetext{
*Corresponding author.
} 
to graphite as described in Ref. [5]. Forced light scattering (FLS, [2]) used two $20 \mathrm{fs}$ pulses of a Ti:sapphire laser to generate a transient grating in the solution of HITCI in ethylene glycol. The delay between the pulses was varied and the grating intensity was monitored as a function of delay by off-resonant probing with $\mathrm{Ar}^{+}$-laser light (Fig. 2). Raman data are used here to model the FLS experiment.

Making the Condon approximation and assuming the low temperature limit, the vibrational contribution to the broadening of the absorption and fluorescence can be calculated (e.g. [3]). It is readily shown, that the spectral density $\rho(\omega)$ is related to the resonance Raman spectrum $I_{R}(\omega)$ by

$$
\rho(\omega) \propto I_{R}\left(\omega ; \omega_{P}\right)\left|\chi^{(1)}\left(\omega_{P}\right)-\chi^{(1)}\left(\omega_{P}-\omega\right)\right|^{-2}
$$

where $\omega_{P}$ is the excitation laser frequency and $\chi^{(1)}$ is the complex linear susceptibility. The imaginary part of $\chi^{(1)}$ is given by the absorption and the real part is obtained by Kramers-Kronig transformation.

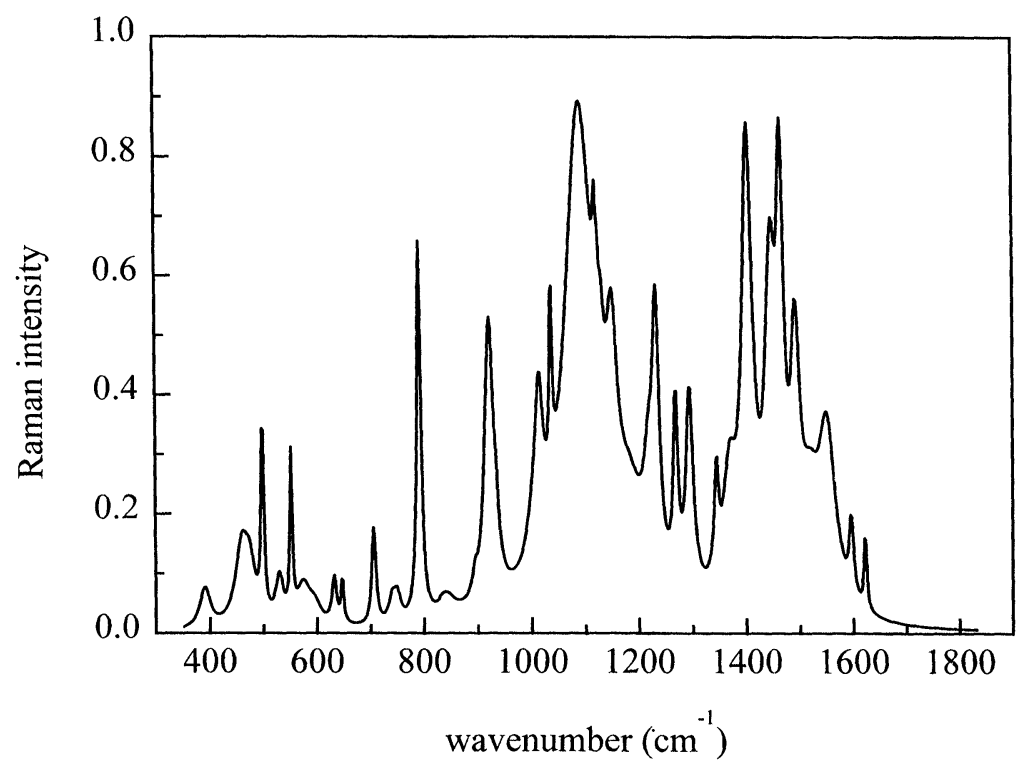

FIGURE 1 Resonance Raman spectrum of HITCI adsorbed on graphite (Raman lines of graphite substracted). 


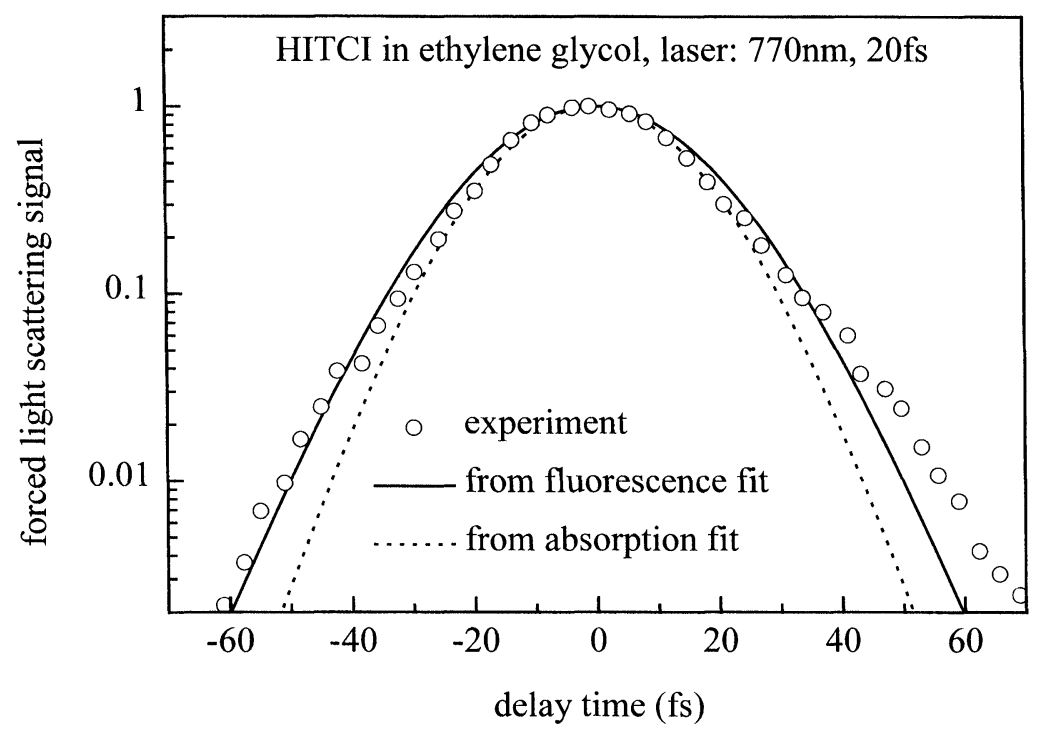

FIGURE 2 FLS signal as function of delay between femtosecond laser pulses.

The line broadening function describing polarization decay is given by

$$
g(t)=\int_{0}^{\infty} d \omega \rho(\omega)\left[\operatorname{coth}\left(\frac{\hbar \omega}{2 k T}\right)(1-\cos \omega t)-i(\omega t-\sin \omega t)\right] .
$$

Figure 3 shows fits to absorption and fluorescence line shapes using the Raman spectrum. Vibrational modes below $300 \mathrm{~cm}^{-1}$ known from pump-probe experiments [4] have been included for completeness. A nice fit to the fluorescence spectrum is obtained by modelling solutesolvent interaction with a single overdamped Brownian oscillator (modulation strength $60 \mathrm{THz}$, correlation time $100 \mathrm{fs}$ ). A fit to the absorption is not possible. The hump on the blue side of the absorption band requires a larger vibrational contribution which leads to large discrepancy in the red cut-off of the absorption. The complete line broadening function obtained from line shape analysis is used to model the FLS data. The fluorescence based fit produces a nice fit even in the wings of the FLS data (Fig. 2).

In conclusion, a single spectral density suffices to describe optical two-level dynamics of rigid molecules. The high frequency part is 


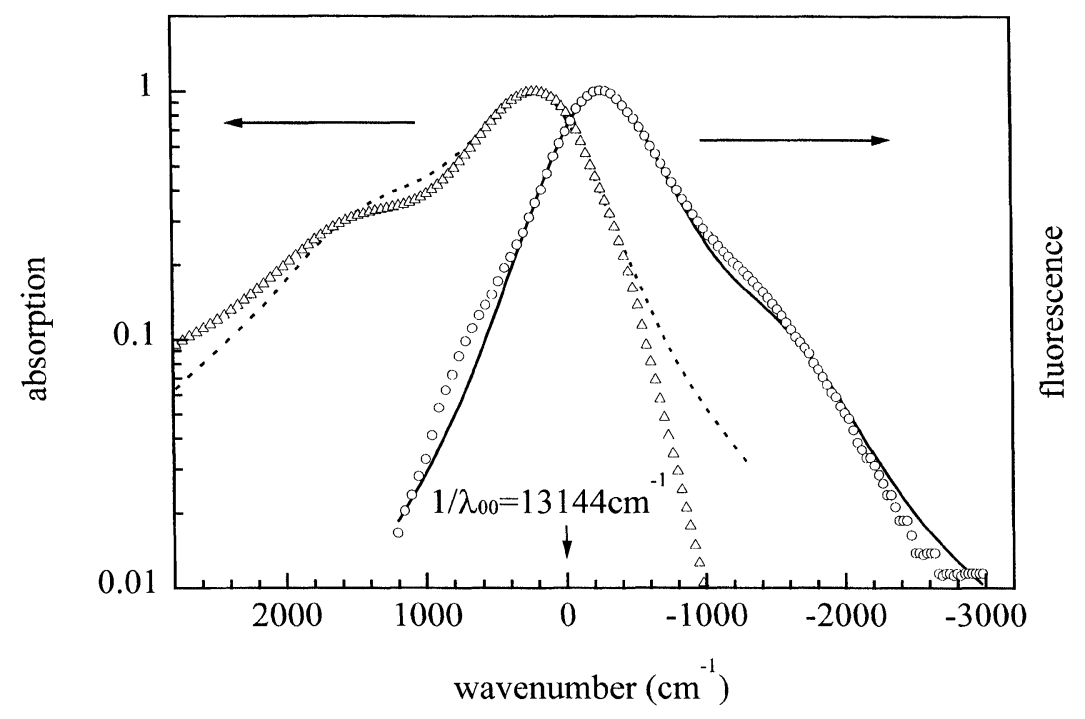

FIGURE 3 Line shape analysis of absorption (triangles) and fluorescence (circles).

hardly distinguishable from the resonance Raman spectrum including proper conversion factors. The low frequency part is difficult to measure by Raman spectroscopy, so femtosecond pump-probe and stimulated photon echo are better suited here.

\section{Acknowledgements}

This work was supported by the Deutsche Forschungsgemeinschaft and the Alexander von Humboldt Stiftung.

\section{References}

[1] Mukamel, S. (1995). Principles of Nonlinear Optical Spectroscopy (Oxford University Press, New York), Chap. 8.

[2] Kummrow, A., Lau, A. and Lenz, K. (1997). Phys. Rev. A, 55, 2310; Kummrow, A., Woggon, S. and Lau, A. (1994). Phys. Rev. A, 50, 4264.

[3] Ashworth, S. H., Kummrow, A. and Lenz, K. (1997). J. Raman Spectrosc., 28, 537.

[4] Vöhringer, P., Westervelt, R. A., Yang, T.-S., Arnett, D. C., Feldstein, M. J. and Scherer, N. F. (1995). J. Raman Spectrosc., 26, 535.

[5] Kagan, M. R. and McCreery, R. L. (1994). Anal. Chem., 66, 4159. 